\title{
Resultado quirúrgico entre cirugía conservadora de mama más injerto graso inmediato versus cirugía conservadora en pacientes con tumores de mama
}

\author{
Surgical result between breast conserving surgery plus immediate \\ fat graft versus conservative surgery in patients with breast tumors
}

Iván González Jiménez, ${ }^{*}$ Cynthia Clarissa Castro Castro, ${ }^{*}$ Roberto Carlos Arenas Mejía*

\begin{abstract}
Palabras clave:
Cáncer de mama, injerto, cirugía conservadora, retracción, dolor.
\end{abstract}

Key words: Breast cancer, graft, conservative surgery, retraction, pain.

\section{RESUMEN}

Introducción: La reconstrucción de la mama ha cobrado importancia; la combinación de un procedimiento oncológico y estético en una sola operación ofrece excelentes resultados. Existen diferentes técnicas que pueden ser útiles, como la aplicación de injerto autógeno. Material y métodos: Se elaboró un estudio de casos y controles que incluyó mujeres mayores de 18 años con tumores de mama uni o bilaterales con tamaño menor a $5 \mathrm{~cm}$, BIRADS IV y $\mathrm{V}$, que requirieron de tratamiento quirúrgico. El grupo de casos estuvo formado por pacientes sometidas a cirugía conservadora de la mama más injerto graso autógeno (CIA), y el grupo control, por mujeres a las que únicamente se les realizó cirugía conservadora de la mama (CCM). Se registró la incidencia de las complicaciones y se evaluó la intensidad del dolor mediante la escala visual análoga. Resultados: La incidencia global de complicaciones ( $\sin$ considerar el dolor) fue $29.2 \%$; en el grupo de CIA fue $12.5 \%$, en el grupo de CCM fue $37.5 \%$. La presencia de dolor a las 24 horas postquirúrgicas fue más frecuente en CCM que en CIA $(81.2 \%[n=13]$ versus $62.5 \%[n=5])$, sin ser estadísticamente diferente $(\mathrm{p}=0.36)$. A los siete días, $25 \%$ de las pacientes $(n=4)$ del grupo CCM presentaban dolor, y del grupo CIA, solamente una $(12.5 \%)(p=$ 0.63). Al finalizar el seguimiento, una paciente de CCM persistió con dolor (6.2\%). El 19\% de las pacientes con CCM desarrollaron retracción de la herida, una de ellas hematoma y otra un seroma. Conclusiones: La CIA es un procedimiento seguro que puede garantizar el mismo resultado quirúrgico que la CCM, pero se puede acompañar de un mejor resultado estético.

\section{ABSTRACT}

Introduction: Breast reconstruction has gained importance, with the combination of an oncological and aesthetic procedure in a single surgery offering excellent results. There are different techniques that can be used, such as the insertion of an autologous graft. Material and methods: A cases-control study was conducted, which included women $>18$ years, with unilateral or bilateral breast tumors $<5$ $\mathrm{cm}$, BIRADS IV and V, that required surgical treatment. The cases group were patients undergoing breast-conserving surgery (BCS) with autologous fat grafts, and the control group were those only undergoing conservative breast surgery (CBS). The incidence of complications was recorded, and the pain intensity was evaluated with the visual analogue scale (EVA). Results: The overall incidence of complications (not considering pain) was $29.2 \%: 12.5 \%$ in the BCS group, and $37.5 \%$ in the CBS group. The presence of pain 24 hours after surgery was more frequent in the CBS group than in the BCS one $(81.2 \%$ [ $n=13]$ versus $62.5 \%$ $[n=5])$, with no statistical difference $(p=.36)$. On the seventh day, $25 \%(n=4)$ of the patients in CBS presented pain, and only one in the BCS group did $(12.5 \%)(p=.63)$. At the end of the study, only one patient in the CBS group persisted with pain (6.2\%), 19\% of patients with CBS developed wound retraction, one hematoma, and another one a seroma. Conclusions: Autologous graft surgery is a safe procedure that guarantees the same surgical outcome as CBS, but with a better aesthetic result.
* Servicio de Cirugía Oncológica, Unidad especialidad Hospital de Especialidades (UMAE-HE), Centro Médico Nacional de Occidente (CMNO), Instituto Mexicano del Seguro Social (IMSS), Guadalajara, Jalisco, México.

Recibido: 01/01/2015 Aceptado: 01/05/2015

\section{INTRODUCCIÓN}

$\mathrm{E}_{\mathrm{m}}^{\prime}$ cáncer de mama es la neoplasia maligna más común en las mujeres estadounidenses y se encuentra en segundo lugar de mortalidad por cáncer, sólo por debajo del cáncer de pul- món. ${ }^{1}$ En México, el cáncer de mama es un importante problema de salud pública que con el tiempo ha incrementado en número de casos y mortalidad. A partir de 2006, ocupa el primer lugar de causa de muerte por neoplasia maligna en mujeres de 25 años o más y ha desplazado 
de esta posición al cáncer cervicouterino. En los últimos años, las instituciones del sector salud han hecho un esfuerzo para informar a la población sobre los métodos de prevención y diagnóstico temprano, haciendo énfasis en la autoexploración mamaria y el estudio de mamografía para las mujeres con edad superior a los 40 años. ${ }^{2,3}$

Aunque el cáncer de mama se ha documentado desde hace más de 5,000 años, como se ha visto en el papiro de Edwin Smith, el tratamiento quirúrgico para esta enfermedad podría describirse como primitivo. En la era preanestésica, el tratamiento quirúrgico consistía en cauterización del tumor para la enfermedad expuesta, ulcerada y localmente avanzada. La mayoría de las complicaciones resultaba en septicemia y muerte. ${ }^{4,5}$ La gran mayoría de operaciones para cáncer de mama en las primeras décadas del siglo XX empleó la mastectomía radical modificada por Meyer. Su operación, publicada unos días después que la de Halsted, incluía la resección del músculo pectoral menor. La cirugía de Halsted-Meyer fue mejor conocida durante la segunda mitad del siglo XX como la mastectomía radical clásica de Halsted..$^{5-7}$ En la actualidad, la reconstrucción de la mama ha cobrado importancia debido a mayores expectativas de las pacientes. Hay un mayor conocimiento de que la reconstrucción inmediata puede combinar procedimientos oncológicos y estéticos en una sola cirugía con excelentes resultados. ${ }^{8-10}$ Actualmente, la cirugía de mama se realiza por cirujanos con adiestramiento oncoplástico que pueden ofrecer reconstrucción inmediata con beneficios oncológicos, estéticos y económicos. ${ }^{11,12}$ Existen diferentes técnicas para lograr una mejoría estética, como la reducción mamaria, la rotación de colgajos pediculados, reacomodo mamario y la aplicación de injerto autógeno. ${ }^{13,14}$

La interpretación mamográfica posterior a la cirugía conservadora de mama (CCM) y radioterapia se dificulta frecuentemente por la formación de cicatrices, deformidad del contorno de la mama, engrosamiento de los ligamentos de Cooper, engrosamiento de la piel y calcificaciones ${ }^{15,16}$ Estudios previos han mostrado que estos cambios comprome- ten la sensibilidad de la mamografía para la posterior detección del cáncer de mama. ${ }^{17,18}$ La CCM comúnmente resulta en defectos de tejidos blandos que son un reto para la reconstrucción, especialmente en pacientes que han recibido radioterapia adyuvante. Estas mujeres a menudo tiene pocas opciones de reconstrucción debido a que los implantes sintéticos comerciales no son utilizables en reconstrucción parcial de la mama, mientras que los procedimientos con colgajos pueden estar asociados con mayor morbilidad en adición al costo. Dados estos retos, los cirujanos continúan en la búsqueda de opciones reconstructivas para enfermas con CCM. Hay buena cantidad de evidencia de que el injerto graso autógeno puede ser útil en el tratamiento de los defectos de mama. ${ }^{15}$ La transferencia de grasa autógena es un procedimiento ampliamente utilizado en cirugía plástica para fines estéticos y reconstructivos. Es comúnmente utilizado en cirugía facial, pero se ha incrementado su uso para restaurar el contorno, incrementar el volumen y mejorar la piel irradiada en la mama, particularmente en casos del tratamiento CCM. ${ }^{17,18}$ Las complicaciones de los injertos grasos, como la necrosis grasa, formación de quistes y calcificaciones, rara vez son causa de morbilidad significativa. ${ }^{17}$ Anteriormente, existía la preocupación de que las calcificaciones postoperatorias de los injertos de grasa pudieran comprometer la detección del cáncer de mama; sin embargo, The American Society of Plastic Surgeons (ASPS) refiere que hasta ahora "no parece haber ninguna interferencia del injerto graso con la detección del cáncer de mama". ${ }^{17}$ El objetivo de este estudio fue comparar el resultado quirúrgico entre la CCM y la cirugía conservadora de mama más injerto graso autógeno $(\mathrm{CIA})$ en pacientes con tumores de mama.

\section{MATERIAL Y MÉTODOS}

Se realizó un estudio de cohorte prospectivo en el Departamento de Oncológica-Quirúrgica, Unidad Médica de Alta Especialidad Hospital de Especialidades (UMAE-HE), Centro Médico Nacional de Occidente (CMNO), Instituto Mexicano del Seguro Social (IMSS), en Guadalajara, Jalisco, durante el periodo 
comprendido entre marzo de 2014 y enero de 2015. Incluyó pacientes con tumores de mama que cumplieron con los siguientes criterios de selección: mujeres mayores de 18 años con tumores de mama uni o bilaterales $<5 \mathrm{~cm}$, con hallazgos mastográficos BIRADS IV y $\mathrm{V}$, que requirieron de tratamiento quirúrgico. Se excluyeron mujeres con historia de cirugía mamaria ipsilateral previa, cáncer de mama y aquéllas que no aceptaron participar en el estudio. El único criterio de eliminación fue las pérdidas en el seguimiento. La cohorte de exposición estuvo formada por aquellas pacientes que se sometieron a CIA, y la cohorte no expuesta, por aquéllas que fueron únicamente sometidas a CCM. Proporciones con un nivel de confianza del 95\%, un poder de la muestra del $80 \%$. El total del tamaño de la muestra fue de 14 pacientes $+20 \%$ de pérdidas al seguimiento, 16 personas por grupo. Las variables dependientes analizadas fueron complicaciones inmediatas y complicaciones tempranas. La variable independiente, CCM más $\mathrm{ClA}$; y las intervinientes, edad, tamaño de la lesión, relación mama/tumor, tipo histológico, BIRADS preoperatorio, tamaño de la resección tumoral, obesidad de la paciente, diabetes mellitus y tabaquismo.

En la CIA, bajo anestesia, ya fuera general o regional, se realizó una incisión en la mama afectada, pudiendo variar en cuanto a la orientación y trayecto según criterio del cirujano



o características de la paciente y tamaño del tumor. Se realizó resección del tumor con un margen de tejido macroscópicamente sano de aproximadamente $1 \mathrm{~cm}$. El tumor fue retirado por el cirujano oncólogo y el cirujano plástico fue el responsable de la extracción de grasa autógena por liposucción en la zona periumbilical. La grasa autógena se procesó para su injerto en la zona donde se extrajo el tumor por medio de la técnica modificada descrita por Coleman. La CCM fue realizada solamente por el cirujano oncólogo y se llevó a cabo el mismo procedimiento hasta la resección del tumor, con mismo margen y posterior cierre de la herida según conveniencia del cirujano. Las complicaciones a detectar fueron la presencia de hematomas, dolor en el sitio de la herida, infección de la herida quirúrgica, retraso en la cicatrización y retracción de la herida. En ambas cohortes, la evaluación de estas complicaciones se realizó mediante interrogatorio dirigido y exploración física en la primera semana, y después, a las tres y seis semanas (de acuerdo con el protocolo de atención establecido en el manejo postquirúrgico de cirugía de mama). La intensidad del dolor en el sitio quirúrgico se evaluó mediante la escala visual análoga. Del resto de las complicaciones sólo se evaluó si estuvieron presentes o ausentes. Las complicaciones se clasificaron en inmediatas (aquéllas que ocurrieron dentro de las 24 horas posteriores a la cirugía) y tempranas (las que sucedieron 30 días después del procedimiento quirúrgico).

\section{RESULTADOS}

Se estudiaron 24 pacientes, de las cuales ocho se incluyeron en el grupo de casos (CIA) y 16 en el grupo control (CCM). El IMC fue significativamente mayor en el grupo de pacientes con CCM, mientras que el tiempo de tabaquismo fue mayor en el grupo de mujeres con CIA. La edad, la frecuencia de tabaquismo y la presencia de diabetes mellitus no fueron diferentes entre los grupos (Cuadro I). En ambos, se incluyeron pacientes con tumores benignos y malignos de la mama. En cuatro pacientes del grupo de mujeres sometidas a $\mathrm{CIA}$, el resultado correspondió a una mastopatía fibroquística, seguida de carcinoma ductal invasivo (dos pa- 
Cuadro II. Comparación de las características histopatológicas de los tumores de mama entre grupos.

\begin{tabular}{ccc} 
Característica radiológica & $\begin{array}{c}\text { Injerto } \\
\text { autólogo (n) }\end{array}$ & $\begin{array}{c}\text { Cirugía } \\
\text { convencional (n) }\end{array}$ \\
\hline
\end{tabular}

Fibroadenoma Mastopatía fibroquística

Hiperplasia ductal

Carcinoma ductal in situ

Carcinoma ductal invasor

Carcinoma lobulillar invasor

Prueba estadística: $\chi^{2}, \mathrm{p}=0.34$
Cuadro III. Evaluación del dolor a las 24

horas según la escala visual análoga, (\%).

\begin{tabular}{ccc}
$\begin{array}{c}\text { Escala del } \\
\text { dolor }\end{array}$ & $\begin{array}{c}\text { Injerto } \\
\text { autólogo (\%) }\end{array}$ & $\begin{array}{c}\text { Cirugía } \\
\text { convencional (\%) }\end{array}$ \\
\hline 1 & $1(20)$ & $1(7.8)$ \\
2 & $1(20)$ & $3(22.6)$ \\
3 & 0 & $3(22.6)$ \\
4 & $1(20)$ & $3(22.6)$ \\
5 & $1(20)$ & 0 \\
6 & $1(20)$ & $1(7.8)$ \\
7 & 0 & $1(7.8)$ \\
8 & 0 & 0 \\
9 & 0 & $1(7.8)$ \\
10 & 0 & 0
\end{tabular}

cientes); mientras que en seis pacientes del de CCM, el resultado fue carcinoma ductal invasivo y mastopatía fibroquística. Las diferencias en la distribución de los hallazgos histopatológicos entre ambos grupos no fueron estadísticamente significativas ( $p=0.34$ ) (Cuadro II).

En cuanto al tamaño de los tumores extirpados a las pacientes, el promedio fue de $17.4 \pm 9.9 \mathrm{~mm}$. De acuerdo con cada grupo, el tamaño del tumor fue de $17.8 \pm 13.2 \mathrm{~mm}$ en el grupo de injerto graso y $17.1 \pm 8.3$ $\mathrm{mm}$ en el de cirugía convencional. Estas diferencias no fueron significativas $(p=0.83)$. En todas las participantes, la relación mama/ tumor fue favorable. La mediana de volumen de grasa injertado fue de $82.5 \mathrm{~mL}$, con un mínimo de 40 y un máximo de $150 \mathrm{~mL}$. La distribución de los hallazgos radiológicos de acuerdo con la clasificación BIRADS no fue diferente entre grupos $(p=0.96)$. Se registró la incidencia de complicaciones en ambos grupos, así como su temporalidad, definiéndose como "inmediatas" a las complicaciones que ocurrieron dentro de las primeras 24 horas del procedimiento y "tempranas" aquéllas que se presentaron durante el periodo comprendido después de las 24 horas y dentro de los 30 días posteriores al procedimiento. La incidencia global de complicaciones (sin considerar el dolor), fue del $29.2 \%$ : la incidencia de complicaciones en el grupo de CIA fue de $12.5 \%$, mientras que en la cohorte de CCM fue de $37.5 \%$. Estas diferencias no fueron significativas $(p=0.35)$.

La presencia de dolor 24 horas después de la cirugía fue más frecuente en la cohorte de CCM que en la de CIA (81.2\% [n=13] vs. $62.5 \%[n=5])$, sin ser esta diferencia estadísticamente significativa $(p=0.36)$. A los siete días, $25 \%$ de las pacientes $(n=4)$ de la cohorte de CCM presentaban dolor, y del grupo CIA, solamente una $(12.5 \%)(p=0.63)$; al final del periodo de seguimiento, sólo una mujer de la cohorte de CCM persistía con dolor (6.2\%) (Cuadro III). Únicamente se muestran los resultados de la intensidad del dolor correspondiente a la medición de las 24 horas debido a que sólo cuatro pacientes presentaron dolor al día siete (tres del grupo de CCM y una de CIA) y en una mujer del grupo CCM el dolor persistió hasta la tercera semana después del evento quirúrgico (llama la atención que esta persona fue la única que en la medición basal presentaba la mayor intensidad en la escala de dolor).

En el grupo de pacientes que fueron sometidas a CIA, la incidencia de dolor fue de $62.5 \%(n=5)$, versus $81.2 \%(n=13)$ en el de CCM; el dolor persistió a los siete días después de la cirugía en $12.5 \%(n=1)$ de las pacientes de CIA, mientras que en el grupo de CCM, cuatro (25\%) persistieron con dolor; sólo una mujer de este mismo grupo (6.25\%) siguió con dolor a las tres semanas. Exclusivamente en el grupo de CCM, tres enfermas presentaron hematoma, seroma y 


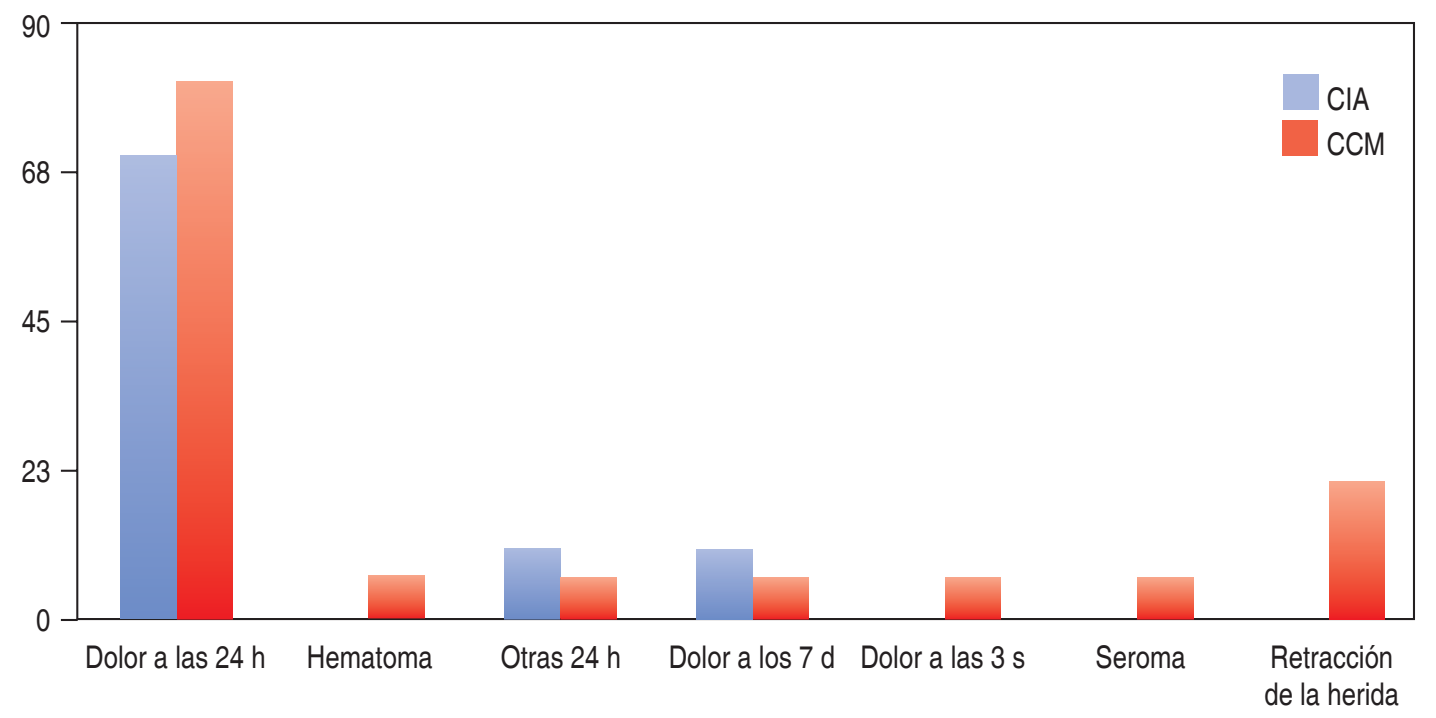

Abreviaturas: $\mathrm{CCM}=$ Cirugía conservadora de mama $. \mathrm{CIA}=$ Cirugía conservadora de mama más injerto graso autógeno. $h=$ Horas. $d=$ Días. $s=$ Semanas.

Figura 1. Tipo de complicaciones entre ambos grupos.

cefalea postpunción, respectivamente. Dentro de este mismo grupo, tres presentaron retracción de la herida quirúrgica (Figura 1). En ninguno de los dos grupos se presentaron casos de infección de la herida quirúrgica, dehiscencia de sutura o retraso en la cicatrización de la herida.

\section{DISCUSIÓN}

En nuestro medio, éste fue el primer estudio que comparó el resultado quirúrgico entre estas dos opciones para reconstrucción inmediata mamaria. Otros autores, como Ihrai y colaboradores, ${ }^{19}$ realizaron un estudio retrospectivo con 100 procedimientos de CIA en 64 pacientes con cáncer de mama, de los cuales sólo en 14 fue utilizado para corregir defectos por la CCM. El tiempo promedio entre la cirugía conservadora y el injerto graso fue de 78.8 meses. Una de las fortalezas de nuestro estudio ${ }^{19}$ fue que el injerto se realizó durante el mismo procedimiento anestésico y mostró un resultado inmediato. La única complicación reportada en la serie ${ }^{19}$ fue un caso de infección del sitio donador. Nuestros resultados muestran que de las pacientes sometidas a CIA, solamente dos casos presentaron complicaciones (cefalea postpunción [no relacionada con el injerto graso] y dolor persistente al día siete del evento quirúrgico).

En la serie de Rose y su grupo, ${ }^{20}$ se evaluaron diversas técnicas de reconstrucción mamaria sin utilizar injerto graso; la principal complicación postquirúrgica fue el hematoma, que se presentó en 10\% de los casos, seguido de seroma e infección, con un caso respectivamente. En nuestro estudio se observó sólo un caso de hematoma en el sitio quirúrgico en el grupo de pacientes con CCM, mientras que en el grupo de CIA no se presentó ningún caso. Otra complicación evaluada en el estudio de Saarela ${ }^{21}$ fue el dolor, reportado en cinco pacientes y sólo descrito como dolor prolongado, sin especificar cronicidad y/o intensidad. Nuestro estudio evaluó tanto la intensidad como la cronicidad del dolor potsoperatorio, evidenciando una media de dolor potsoperatorio aceptable (tres en la escala visual análoga), con sólo una paciente que lo presentó después de tres semanas de la intervención quirúrgica en la cohorte de pacientes con CCM. Aunque nuestros resultados no mostraron diferencias significativas en cuanto a la incidencia de complicaciones, intensidad del dolor y tipo de complicaciones entre ambas cohortes, el resultado clínico sí fue mejor cuando se realizó la CIA. 


\section{CONCLUSIONES}

La incidencia de complicaciones en el total de las mujeres incluidas en el presente estudio fue de $29.2 \%$. Ninguna de estas complicaciones fue considerada grave $y / o$ que dejara secuelas importantes que afectaran la función de las pacientes. La incidencia de complicaciones en el grupo de CIA fue de $12.5 \%$ mientras que en la cohorte de CCM fue de $37.5 \%$; esta diferencia no fue estadísticamente significativa; sin embargo, sí tiene un impacto clínico favorable a favor de la CIA. La presencia de dolor 24 horas después de la cirugía fue más frecuente en la cohorte de CCM que en la de CIA (81.2\% versus $71 \%$ ), así como la persistencia del dolor a los siete días y tres semanas después del procedimiento quirúrgico. El resultado anterior puede reflejar un mayor beneficio clínico $y$, sobre todo, de bienestar para la paciente cuando se prefiere la CIA en lugar de la CCM. Sin embargo, es necesario para demostrar lo anterior que en futuros estudios se mida el impacto de la cirugía sobre la satisfacción y la calidad de vida de las pacientes.

La complicación más frecuente en ambos grupos fue el dolor; sin embargo, la incidencia fue menor en las mujeres sometidas a CIA. Sólo en el grupo de CCM, tres pacientes presentaron hematoma, seroma y cefalea postpunción, respectivamente. Aunque no se encontró una diferencia significativa en el tipo de complicación entre los dos grupos; sí llama la atención que tres pacientes de las 16 sometidas a CCM presentaron retracción de la herida quirúrgica, lo cual puede comprometer el resultado estético del procedimiento. En ninguno de los dos grupos se presentaron casos de infección de la herida quirúrgica, dehiscencia de sutura o retraso en la cicatrización de la herida. Con todo esto, podemos afirmar que la $\mathrm{CIA}$ es efectiva y segura para la reconstrucción mamaria en mujeres con tumores de mama, por lo que se debe proponer como una alternativa que puede ayudar a obtener un mejor resultado quirúrgico y estético de las pacientes sometidas a CCM.

\section{REFERENCIAS}

1. American Cancer Society. Cancer Facts and figures 2012. Atlanta ACS, 2012 [Acceso marzo de 2014]. Disponible en: http://www.cancer.org
2. Early Breast Cancer Trialists' Collaborative Group (EBCTCG). Effects of chemotherapy and hormonal therapy for early breast cancer on recurrence and 15-year survival: an overview of the randomized trials. Lancet. 2005; 365: 1687-1717.

3. Siegel R, Ward E, Brawley O, Jemal A. Cancer statistics, 2011: the impact of eliminating socioeconomic and racial disparities on premature cancer deaths. CA Cancer J Clin. 2011; 61: 212-236.

4. Secretaría de Salud. Perfil epidemiológico de los tumores malignos en México. México: Secretaría de Salud; 2011.

5. Winchester D, Trabanino L, Lopez M. The evolution of surgery for breast cancer. Surg Oncol Clin N Am. 2005; 14: 479-498.

6. Harvey A. Early contributions to the surgery of cancer: William S. Halsted, Hugh H. Young and John G. Clark. Johns Hopkins Med J. 1974; 135: 399-417.

7. Patey D. A review of 146 cases of carcinoma of the breast operated on between 1930 and 1943. Br J Cancer. 1967; 21: 260-269.

8. Madden J. Modified radical mastectomy. Surg Gynecol Obstet. 1965; 121: 1221-1230.

9. Robinson GN, van Heerden JA, Payne WS, Taylor WF, Gaffey TA. The primary surgical treatment of carcinoma of the breast: a changing trend toward modified radical mastectomy. Mayo Clin Proc. 1976; 51: 433-442.

10. Morrow M, Strom EA, Bassett LW, Dershaw DD, Fowble B, Giuliano A, et al. Standard for breast conservation therapy in the management of invasive breast cancer. CA Cancer J Clin. 2002; 52: 277-300.

11. Van Dongen JA, Voogd AC, Fentiman IS, Legrand C, Sylvester RJ, Tong D, et al. Long-term results of a randomized trial comparing breast-conserving therapy with mastectomy: European Organization for Research and Treatment of Cancer 10801 trial. J Natl Cancer Inst. 2000; 92: 1143-1150.

12. Fisher B, Anderson S, Bryant J, Margolese RG, Deutsch $M$, Fisher ER, et al. Twenty-year follow-up of a randomized trial comparing total mastectomy, lumpectomy, and lumpectomy plus irradiation for the treatment of invasive breast cancer. N Engl J Med. 2002; 347: 1233-1234.

13. Veronesi U, Cascinelli N, Mariani L, Greco M, Saccozzi $R$, Luini A, et al. Twenty-year follow-up of a randomized study comparing breast-conserving surgery with radical mastectomy for early breast cancer. $N$ Engl J Med. 2002; 347: 1227-1232.

14. Association of Breast Surgery at BASO; Association of Breast Surgery at BAPRAS; Training Interface Group in Breast Surgery, Baildam A, Bishop H, Boland G, et al. Oncoplastic breast surgery-a guide to good practice. Eur J Surg Oncol. 2007; 33 Suppl 1: S1-23.

15. Cárdenas-Sánchez J, Bargalló-Rocha E, Erazo-Valle A. Consenso mexicano sobre diagnóstico y tratamiento del cáncer mamario. Quinta revisión. México: Elsevier; 2013. pp. 18-20.

16. Lohsiriwat V, Curigliano G, Rietjens M, Goldhirsch A, Petit JY. Autologous fat transplantation in patients with breast cancer: "silencing" or "fueling" cancer recurrence? Breast. 2011; 20: 351-357.

17. Pearl RA, Leedham SJ, Pacifico MD. The safety of autologous fat transfer in breast cancer: lessons from stem cell biology. J Plast Reconstr Aesthet Surg. 2012; 65: 283-288. 
18. Breest Smallenburg V, Duijm LE, Voogd AC, Jansen FH, Louwman MW. Mammographic changes resulting from benign breast surgery impair breast cancer detection at screening mammography. Eur J Cancer. 2012; 48: 2097-2103.

19. Ihrai T, Georgiou C, Machiavello JC, Chignon-Sicard B, Figl A, Raoust I, et al. Autologous fat grafting and breast cancer recurrences: retrospective analysis of a series of 100 procedures in 64 patients. J Plast Surg Hand Surg. 2013; 47: 273-275.

20. Rose M, Manjer J, Ringberg A, Svensson H. Surgical strategy, methods of reconstruction, surgical margins and postoperative complications in oncoplastic breast surgery. Eur J Plast Surg. 2014; 37: 205-214.
21. Saarela AO, Kiviniemi HO, Rissanen TJ, Haukipuro K, Kaarela O. Cosmetic results after wire-guided biopsy of benign breast lesions. J Am Coll Surg. 1998; 187: 610-615.

\author{
Correspondencia: \\ Iván González Jiménez \\ Privada Río Tizapan Núm. 1678, \\ Col. Atlas Guadalajara, Jalisco. \\ Tel: 3336358126 \\ Cel.: 3331676585 \\ E-mail: cirujano.ivan@gmail.com
}

